\title{
Automated Trimethyl Sulfonium Hydroxide Derivatization Method for High-Throughput Fatty Acid Profiling by Gas Chromatography-Mass Spectrometry
}

\author{
Paul Gries ${ }^{1,2}$, Atul Singh Rathore 1,2 ${ }^{\mathbb{D}}$, Xiyuan Lu ${ }^{1,2}$, Jennifer Chiou ${ }^{1,2}$, Yen Bao Huynh ${ }^{1,2}$, Alessia Lodi ${ }^{1,2}$ and \\ Stefano Tiziani $1,2,3, *$ (D) \\ 1 Department of Nutritional Sciences, College of Natural Sciences, The University of Texas at Austin, \\ Austin, TX 78712, USA; gries.paul@utexas.edu (P.G.); atulsr2001@utexas.edu (A.S.R.); xlu@utexas.edu (X.L.); \\ jennifer.chiou@austin.utexas.edu (J.C.); yen.hby@utexas.edu (Y.B.H.); alessialodi@utexas.edu (A.L.) \\ 2 Department of Pediatrics, Dell Medical School, The University of Texas at Austin, Austin, TX 78723, USA \\ 3 Department of Oncology, Dell Medical School, LiveSTRONG Cancer Institutes, The University of Texas at \\ Austin, Austin, TX 78723, USA \\ * Correspondence: tiziani@austin.utexas.edu; Tel.: +1-512-495-4706
}

Citation: Gries, P.; Rathore, A.S.; Lu, X.; Chiou, J.; Huynh, Y.B.; Lodi, A.; Tiziani, S. Automated Trimethyl Sulfonium Hydroxide Derivatization Method for High-Throughput Fatty Acid Profiling by Gas

Chromatography-Mass Spectrometry. Molecules 2021, 26, 6246. https:// doi.org/10.3390/molecules26206246

Academic Editors: Angelo

Antonio D'Archivio and

Alessandra Biancolillo

Received: 29 September 2021

Accepted: 12 October 2021

Published: 15 October 2021

Publisher's Note: MDPI stays neutral with regard to jurisdictional claims in published maps and institutional affiliations.

Copyright: (c) 2021 by the authors. Licensee MDPI, Basel, Switzerland. This article is an open access article distributed under the terms and conditions of the Creative Commons Attribution (CC BY) license (https:/ / creativecommons.org/licenses/by/ $4.0 /)$.

\begin{abstract}
Fatty acid profiling on gas chromatography-mass spectrometry (GC-MS) platforms is typically performed offline by manually derivatizing and analyzing small batches of samples. A GCMS system with a fully integrated robotic autosampler can significantly improve sample handling, standardize data collection, and reduce the total hands-on time required for sample analysis. In this study, we report an optimized high-throughput GC-MS-based methodology that utilizes trimethyl sulfonium hydroxide (TMSH) as a derivatization reagent to convert fatty acids into fatty acid methyl esters. An automated online derivatization method was developed, in which the robotic autosampler derivatizes each sample individually and injects it into the GC-MS system in a high-throughput manner. This study investigated the robustness of automated TMSH derivatization by comparing fatty acid standards and lipid extracts, derivatized manually in batches and online automatically from four biological matrices. Automated derivatization improved reproducibility in 19 of 33 fatty acid standards, with nearly half of the 33 confirmed fatty acids in biological samples demonstrating improved reproducibility when compared to manually derivatized samples. In summary, we show that the online TMSH-based derivatization methodology is ideal for high-throughput fatty acid analysis, allowing rapid and efficient fatty acid profiling, with reduced sample handling, faster data acquisition, and, ultimately, improved data reproducibility.
\end{abstract}

Keywords: GC-MS; fatty acid profiling; online automated derivatization; trimethyl sulfonium hydroxide

\section{Introduction}

Fatty acid profiling is a commonly applied analytical methodology in academic research, health care, and industrial production and is used in a variety of applications that span from analyzing metabolic biomarkers to tracking environmental pollutants [1-8]. Advances in robotic platforms and associated software have enabled the automation of many tasks that previously required repetitive and extensive manual labor [9-13]. In addition to reducing sample handling, the integration of automatic sample preparation has improved data reproducibility for large sample batches [13-16]. The development of automated metabolite preparation and profiling methodologies that reduce researcher handling while maintaining or improving data quality are in high demand. Thus, advancements in automated sample preparation methodologies are beneficial to both academic and industrial laboratories.

Gas chromatography-mass spectrometry (GC-MS) platforms are well established for fatty acid analysis and are widely used in lipidomic and metabolomic 
research [8,17-28]. GC-MS analysis of fatty acids requires the derivatization of these analytes into non-polar derivatives, such as fatty acid methyl esters (FAMEs) [29]. This transformation improves sample volatility and subsequent chromatographic separation of the individual fatty acids [30-32]. Lipid researchers use numerous derivatizing agents, and each derivatization process can vary significantly in the number of steps and time required for sample preparation before GC-MS acquisition. Additionally, many derivatization processes involve lengthy incubations at high temperatures that can potentially induce metabolite degradation [33]. Derivatizing reagents, such as $\mathrm{N}, \mathrm{O}$-Bis(trimethylsilyl)trifluoroacetamide (BSTFA), boron trifluoride $\left(\mathrm{BF}_{3}\right)$, and anhydrous hydrogen chloride $(\mathrm{HCl})$, require long heated incubation steps at temperatures as high as $95^{\circ} \mathrm{C}$ [34-36]. Metabolic degradation has been observed at $100^{\circ} \mathrm{C}$. However, fatty acids and their representative FAMEs have been reported to be stable when exposed to temperatures higher than $300{ }^{\circ} \mathrm{C}[33,37,38]$. $\mathrm{HCl}, \mathrm{BF}_{3}$, and potassium hydroxide $(\mathrm{KOH})$ require a second extraction after the initial derivatization [39]. M-(trifluoromethyl)phenyltrimethylammonium hydroxide (TMTFTH) derivatization commonly calls for an overnight incubation before analysis [40]. These additional steps result in a high level of sample handling and time dedicated to prepare samples for fatty acid profiling compared to alternative metabolomic and lipidomic techniques that do not require derivatization. Additionally, without recourse to multiple analytical platforms analyzing samples simultaneously, extensive sample preparation leads to varying incubation times for individual samples. These factors reduce data reproducibility.

The automation of sample preparation is a viable method to eliminate researcher handling-induced variability. Similar approaches in automating sample preparation and derivatization have improved reproducibility in the analysis of sugars, organic acids, and amino acids $[13,15,22,41]$. In this study, we introduce a novel high-throughput automated GC-MS-based methodology that is ideal for uninterrupted fatty acid analysis. Trimethyl sulfonium hydroxide (TMSH), a derivatization reagent with a simple derivatization reaction and minimal sample handling that has been previously applied to fatty acid profiling [42-46], was selected and applied to work in an automated high-throughput manner. Capitalizing on the robotic autosampler attached to a GC-MS system, an automated online derivatization method was developed. The robotic sampler independently derivatizes each sample and injects it in an online high-throughput manner. In the developed automated method, each sample was derivatized immediately before injection in direct comparison with the common practice of manual batch derivatization followed by batch analysis, which results in time discrepancies between derivatization and injection for each sample. This study tested the robustness of the GC-MS method by comparing the automated and manual TMSH derivatization performed in batches or performed on the same set of batches.

\section{Results and Discussion}

\subsection{Adaptation of Method to Automation}

Commonly utilized fatty acid derivatization methods were compared (Figure 1), including the number of handling steps required for each derivatization reagent and the time before the sample is ready for injection and analysis. Several widely used derivatization reagents for fatty acids require extensive handling in the forms of secondary extractions or long heated incubations, such as those required by the standard $\mathrm{KOH}, \mathrm{HCl}$, and $\mathrm{BF}_{3}$ derivatization reactions. 


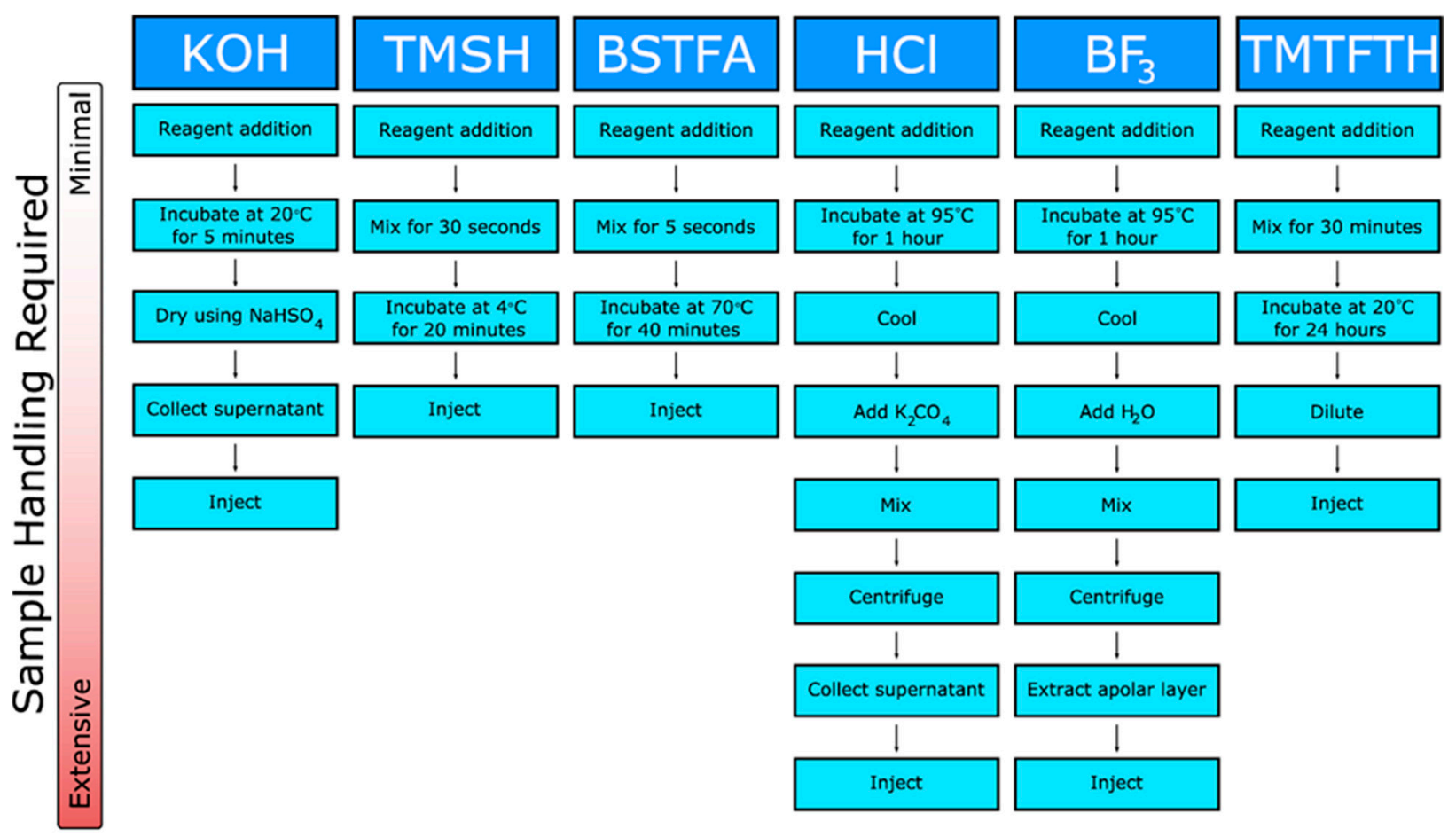

Time Required Before Injection

Figure 1. Time-based comparison of common fatty acid derivatization methodologies. Step-by-step workflow of common fatty acid derivatization techniques reported by various groups. Each independent step in sample preparation is illustrated, and the time required to prepare individual samples increases from left to right across the workflow. Required sample handling increases from top to bottom down each derivatization technique.

Due to the lack of need for a secondary extraction step and the short incubation time and simplicity, the TMSH derivatization method was selected and adapted for the Triplus RSH autosampler through the editor software. TMSH methylation occurs through a base-catalyzed transesterification reaction. Figure 2 shows the mechanism in which TMSH induces O-methylation of the carboxyl group of fatty acids when heated by the pyrolytic conditions of a GC injection port [47-49]. When performed manually, researchers can have a batch of samples ready for analysis within $30 \mathrm{~min}$. However, as the samples are prepared in batches, the length of time each sample is incubated for can vary greatly between the first and last injected samples. Regarding the method outlined above, with batches of eight samples and a $20 \mathrm{~min}$ GC-MS runtime, there is a $160 \mathrm{~min}$ difference in incubation times between the first and last samples analyzed. In contrast, the automated method prepares each sample immediately before injection, and it is programmed to begin preparing each sample during the analysis of the previously injected sample. Automated sample handling ensures a consistent incubation period among all analyzed samples and reduces the variability between samples $[15,22]$. 


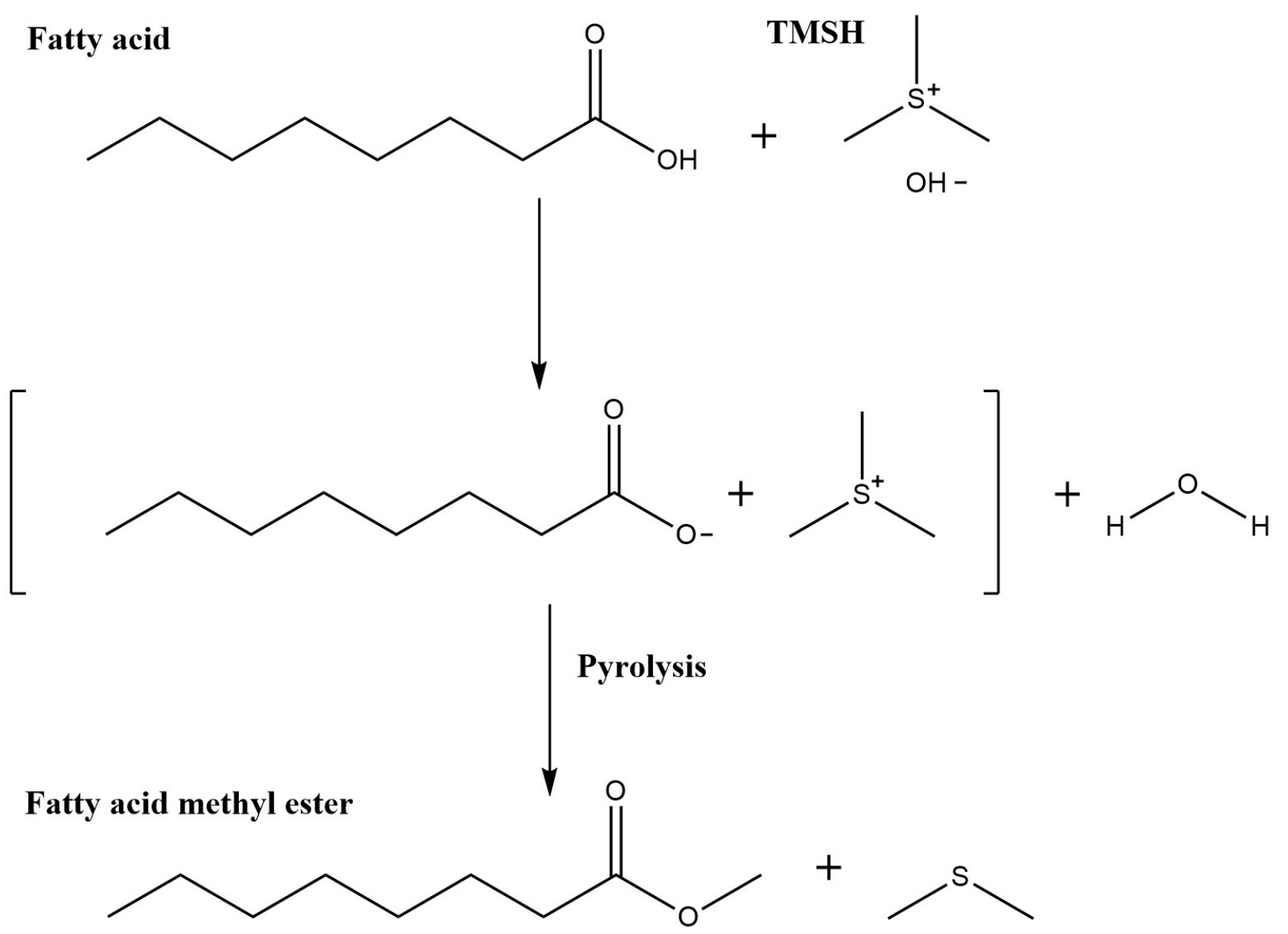

Figure 2. TMSH reaction outline for the conversion of fatty acids into fatty acid methyl esters by base-catalyzed transesterification and pyrolysis.

Moreover, automated derivatization decreases the time needed to analyze large batches of samples. Figure 3 depicts how automated derivatization reduces the time needed to analyze samples. To manually derivatize and analyze 100 samples in the manner presented above would require a minimum of $40 \mathrm{~h}$ just for sample derivatization and acquisition. In comparison, an analysis of 100 samples would take only $34 \mathrm{~h}$ if the autosampler derivatized and injected the samples in an online manner. Automated sample handling produces a $15 \%$ reduction in total time, including handling time by researchers, to derivatize and analyze the samples. A 15\% reduction in analysis time itself may not represent a compelling improvement on its own; however, automated sample derivatization improves several additional aspects of sample handling and data acquisition. Primarily, automation reduces the negative consequences of researcher interaction required to acquire sample data, mainly through decreasing human errors that can be caused by fatigue or variability between researcher efforts. The robotic autosampler does not tire after 10 samples and has a higher consistency in the small tasks needed to prepare samples for analysis. It is important to consider that this methodology, although tested on a GC/single quad MS system, can be easily adapted to any analytical platform that has a programmable robotic autosampler. 


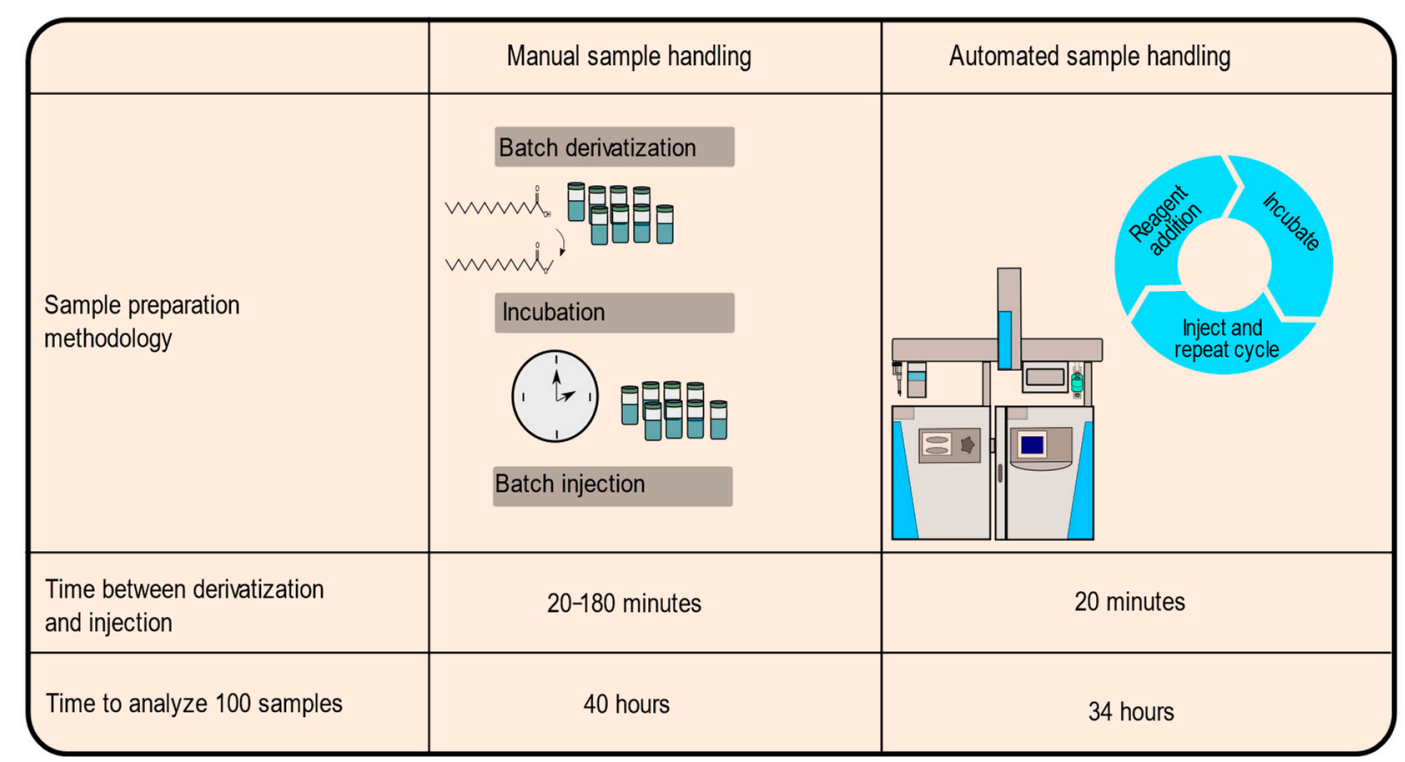

Figure 3. Reduction in time needed for analysis. Time-based comparison of manual and automated TMSH derivatization of fatty acids. Complete analysis of 100 samples manually in 10 batches of 10 samples each would require $40 \mathrm{~h}$ if batches were derivatized as soon as the previous batch finished. Automated derivatization would require $34 \mathrm{~h}$ with continuous derivatization and injection.

\subsection{Automated TMSH Derivatization Test on Fatty Acid Standard Mixture}

To determine the efficiency of the automated TMSH derivatization, a mixture of 33 fatty acid standards was derivatized both manually and automatically. The reproducibility of the fatty acid derivatization is reported by the relative standard deviation (RSD) of each detected and confirmed lipid (Table 1); \%RSD is a common indicator for the reproducibility of metabolomic data $[13,22]$. As previously reported, for a GC-MS analysis, a $\%$ RSD $<20 \%$ is considered acceptable reproducibility, whereas a $\%$ RSD $<10 \%$ is classified as superb reproducibility $[13,20,50]$. All except one of the fatty acid standards were detected and reported with good reproducibility (\%RSD $<20 \%)$. Hexacosanoic acid (C26:0) exhibited a reproducibility of $23.61 \%$ using manual TMSH derivatization methodology. In total, 19 out of the 33 fatty acid standards utilized demonstrated improved reproducibility after automated derivatization when compared directly to manually derivatized standards (Figure 4A). Moreover, 31 out of the 33 fatty acid standards resulted in very high reproducibility ( $\%$ RSD $<15 \%$ ), as shown by the red dotted line (Figure 4 A), after automated derivatization and online injection. Docosahexaenoic (C22:6) and tetracosanoic (C24:0) acids demonstrated a \%RSD greater than $10 \%$ (13.65\% and $12.58 \%$, respectively) following the automated derivatization. Only docosahexaenoic and tetracosanoic acids exhibited worse reproducibility after being derivatized through automation, with no significant differences in reproducibility of the other fatty acid standards. The overall efficacy of automated derivatization is shown in Figure 5A, displaying the trend that automated derivatization has on \%RSD across the fatty acid standards. When the automatically derivatized fatty acid $\%$ RSD values are plotted against the manually derived fatty acids, the trendline slope is less than one, indicating an overall improvement in \%RSD and data reproducibility through automated derivatization. 
Table 1. Compiled relative standard deviations of analyzed free fatty acid standards after manual and automated TMSH derivatization. Improvements in reproducibility are indicated with bolded RSD values.

\begin{tabular}{|c|c|c|c|c|c|c|}
\hline \multirow{2}{*}{ Fatty Acid } & \multirow{2}{*}{ HMDB ID } & \multirow{2}{*}{$\begin{array}{c}\text { Carbons: } \\
\text { Double Bonds }\end{array}$} & \multirow{2}{*}{$\begin{array}{c}\text { FAME Molecular } \\
\text { Mass }\end{array}$} & \multirow{2}{*}{$\begin{array}{c}\text { Quantifier } \\
\text { Ion }(m / z)\end{array}$} & \multicolumn{2}{|c|}{ Standards $n=6$} \\
\hline & & & & & Manual & Automatic \\
\hline Heptanoic acid & HMDB0000666 & $\mathrm{C} 7: 0$ & 144.21 & 73.98 & 7.00 & 5.35 \\
\hline Octanoic acid & HMDB0000482 & $\mathrm{C} 8: 0$ & 158.24 & 73.98 & 8.18 & 5.59 \\
\hline Nonanoic acid & HMDB0000847 & C9:0 & 172.26 & 73.98 & 6.36 & 5.46 \\
\hline Decanoic acid & HMDB0000511 & C10:0 & 186.27 & 73.98 & 4.45 & 2.88 \\
\hline Undecanoic acid & HMDB0000947 & $\mathrm{C} 11: 0$ & 200.29 & 73.95 & 4.57 & 5.73 \\
\hline Dodecanoic acid & HMDB0000638 & $\mathrm{C} 12: 0$ & 214.32 & 73.93 & 7.76 & 6.82 \\
\hline Tridecanoic acid & HMDB0000910 & $\mathrm{C} 13: 0$ & 228.35 & 73.94 & 3.96 & 3.51 \\
\hline $9(Z)$-Tetradecenoic acid & HMDB0002000 & C14:1 & 240.36 & 55.05 & 5.97 & 6.48 \\
\hline Tetradecanoic acid & HMDB0000806 & C14:0 & 242.38 & 73.99 & 4.53 & 3.45 \\
\hline 9(Z)-Pentedecenoic acid & HMDB0029765 & C15:1 & 254.41 & 55.05 & 5.49 & 4.13 \\
\hline Pentadecanoic acid & HMDB0000826 & $\mathrm{C} 15: 0$ & 256.42 & 73.96 & 4.97 & 4.85 \\
\hline 9(Z)-Hexadecenoic acid & HMDB0003229 & C16:1 & 268.41 & 55.05 & 4.61 & 4.81 \\
\hline Hexadecanoic acid & HMDB0000220 & $\mathrm{C} 16: 0$ & 270.45 & 73.95 & 5.56 & 4.24 \\
\hline 10(Z)-Heptadecenoic acid & HMDB0060038 & $\mathrm{C} 17: 1$ & 282.46 & 55.05 & 3.94 & 3.33 \\
\hline Heptadecanoic acid & HMDB0002259 & C17:0 & 284.46 & 73.96 & 6.03 & 4.91 \\
\hline$(6 Z, 9 Z, 12 Z)$-Octadecatrienoic acid & HMDB0003073 & C18:3 & 292.44 & 292.18 & 2.37 & 3.21 \\
\hline$(9 Z, 12 Z)$-octadecadienoic acid & HMDB0000673 & $\mathrm{C} 18: 2$ & 294.45 & 294.18 & 4.33 & 4.90 \\
\hline 9(Z)-octadecenoic acid & HMDB0000207 & C18:1 & 296.68 & 296.20 & 1.55 & 5.57 \\
\hline Octadecanoic acid & HMDB0000827 & C18:0 & 298.48 & 73.98 & 3.50 & 3.57 \\
\hline Nonadecanoic acid & HMDB0000772 & C19:0 & 312.53 & 73.96 & 4.11 & 2.71 \\
\hline$(5 Z, 8 Z, 11 Z, 14 Z)$-Icosatetraenoic acid & HMDB0001043 & C20:4 & 318.49 & 318.20 & 6.26 & 4.57 \\
\hline$(5 Z, 8 Z, 11 Z)$-Eicosatrienoic acid & HMDB0010378 & $\mathrm{C} 20: 3$ & 320.51 & 320.20 & 10.91 & 9.47 \\
\hline$(11 Z, 14 Z)$-Eicosadienoic acid & HMDB0005060 & $\mathrm{C} 20: 2$ & 322.51 & 322.25 & 4.56 & 9.58 \\
\hline 13(Z)-Eicosenoic acid & HMDB0035159 & C20:1 & 324.50 & 324.19 & 2.10 & 5.45 \\
\hline Eicosanoic acid & HMDB0002212 & C20:0 & 326.56 & 73.99 & 5.07 & 4.57 \\
\hline Heinecosanoic acid & HMDB0002345 & C21:0 & 340.58 & 73.97 & 4.30 & 2.60 \\
\hline $\begin{array}{c}(4 Z, 7 Z, 10 Z, 13 Z, 16 Z \\
\text { 19Z)-Docosahexaenoic acid }\end{array}$ & HMDB0002183 & C22:6 & 342.52 & 79.06 & 5.51 & 13.65 \\
\hline (13Z)-Docosenoic acid & HMDB0002068 & $\mathrm{C} 22: 1$ & 352.57 & 55.05 & 2.46 & 6.35 \\
\hline Docosanoic acid & HMDB0000944 & $\mathrm{C} 22: 0$ & 354.59 & 74.00 & 5.97 & 9.16 \\
\hline Tricosanoic acid & HMDB0001160 & C23:0 & 368.62 & 74.00 & 2.76 & 3.70 \\
\hline (15Z)-Tetracosenoic acid & HMDB0002368 & C24:1 & 379.62 & 55.06 & 7.33 & 1.99 \\
\hline Tetracosanoic acid & HMDB0002003 & C24:0 & 381.36 & 73.99 & 7.49 & 12.58 \\
\hline Hexacosanoic acid & HMDB0002356 & $\mathrm{C} 26: 0$ & 410.69 & 73.96 & 23.61 & 4.27 \\
\hline
\end{tabular}

\subsection{Validation of the Automated TMSH Derivatization with Biological Samples}

After the automated TMSH derivatization method was successfully applied to the analysis of fatty acid standards, we tested this methodology using common biological matrices (Figures $4 \mathrm{~B}$ and $5 \mathrm{~B}$ ). Fatty acid profiling has numerous applications, such as disease detection, microbiome identification, and pollution monitoring [2,28,51,52]. The non-polar extracted fractions of HepG2, DU145, U937 cell samples, and FBS samples were analyzed. Figure 6 illustrates representative chromatograms for each sample type. The \%RSD values are reported to represent reproducibility and are compared between manual and automated TMSH derivatization (Table 2). The \%RSD values show a direct comparison for the \%RSD values of each fatty acid and the trends that automation has upon reproducibility (Figures $4 \mathrm{~B}$ and $5 \mathrm{~B}$ ). Upon automation, the reproducibility of 17 out of the 33 fatty acids in the FBS samples improved. In terms of cell samples, automated derivatization improved the reproducibility of 9 fatty acids in the liver cancer cell line HepG2, 19 in the prostate cancer line DU145, and 15 in the acute myeloid leukemia line U937. Polyunsaturated fatty acids displayed higher variance than mono-unsaturated and saturated fatty acids in biological samples. Icosatetraenoic acid (C20:4) and eicosatrienoic acid (C20:4) demonstrated some of the highest variances in automated and manual TMSH derivatization when analyzed in DU145. Automation improved reproducibility by increasing \%RSD from $71.96 \%$ to $28.56 \%$ and $40.73 \%$ to $20.70 \%$. Overall, there is a definitive trend of improved \%RSD values across all biological matrices, as shown in Figure 5B. The trendlines generated by the ratios of automated derivatization \%RSD values over the manually derivatized \%RSD values have slopes less than one, indicating that automated 
TMSH derivatization improved reproducibility over those of manually derivatized fatty acids in both standard mixture and biological samples.
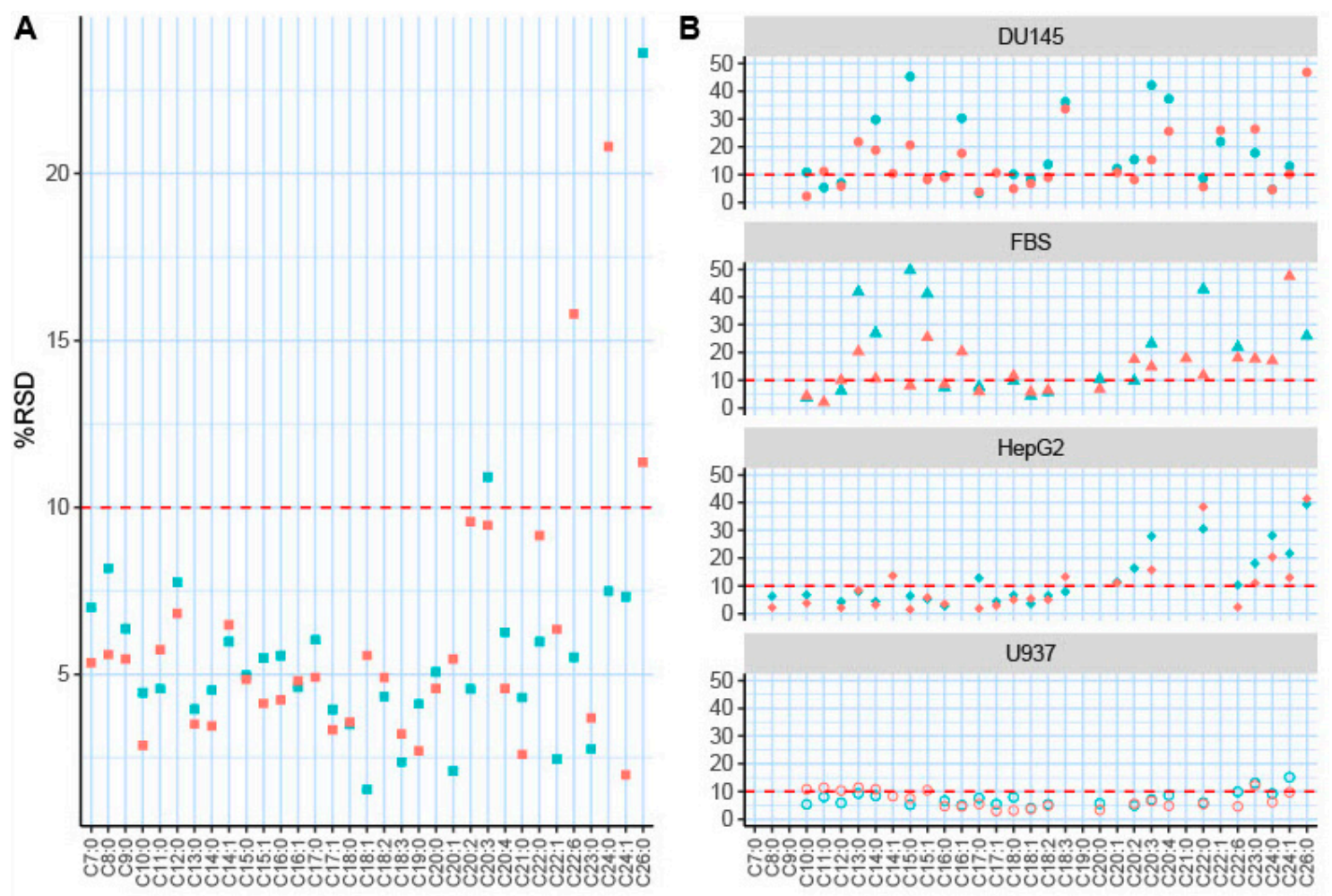

Derivatization " Automated " Manual

Figure 4. The \%RSD comparison for fatty acid derivatization methods. Plot comparison of all \%RSD values for each derivatization technique and each biological matrix. The red line across the plot at 15 represents a commonly accepted cutoff for highly reproducible metabolomics data. (A) The \%RSD values for the 33 fatty acid standards utilized to validate the method. (B) The reproducibility of each fatty acid in the following biological matrices: human prostate cancer cell line DU145; fetal bovine serum (FBS); human liver cancer cell line HepG2; and U937, a human myeloid leukemia cell line. 


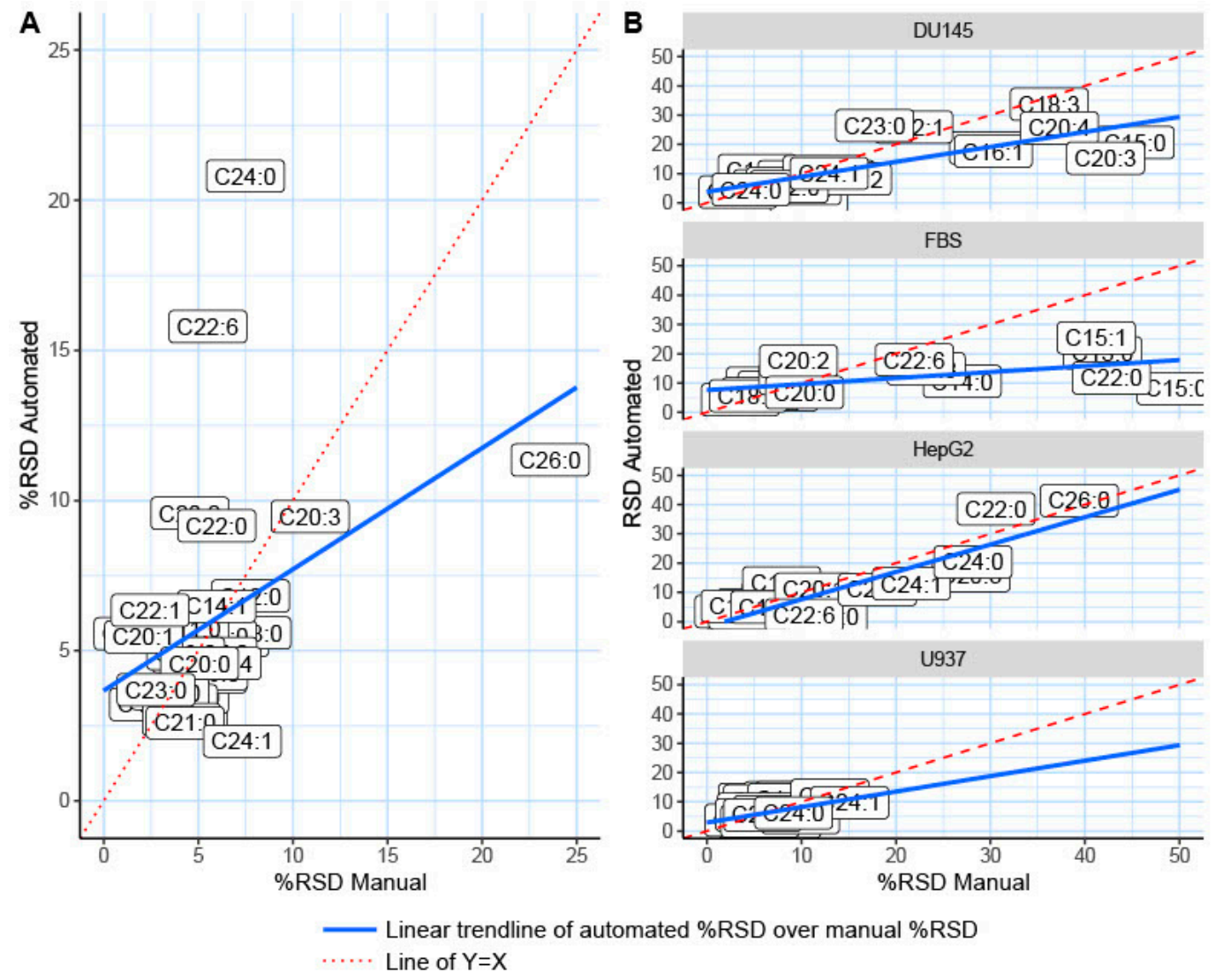

Figure 5. Automated derivatization improves \%RSD trend (A). The ratio of the \%RSD from the automatically derivatized fatty acid standards plotted over the \%RSD of the manually derivatized fatty acid standards. The dotted red line represents a slope of 1 across the plot. Fatty acids that lie beneath that line demonstrate improved automation \%RSD values. The blue line is a linear trendline derived from the data points. (B) The fatty acid derivatization ratios for all biological matrices (human prostate cancer cell line DU145, FBS, human liver cancer cell line HepG2, and human myeloid leukemia cell line) were tested.

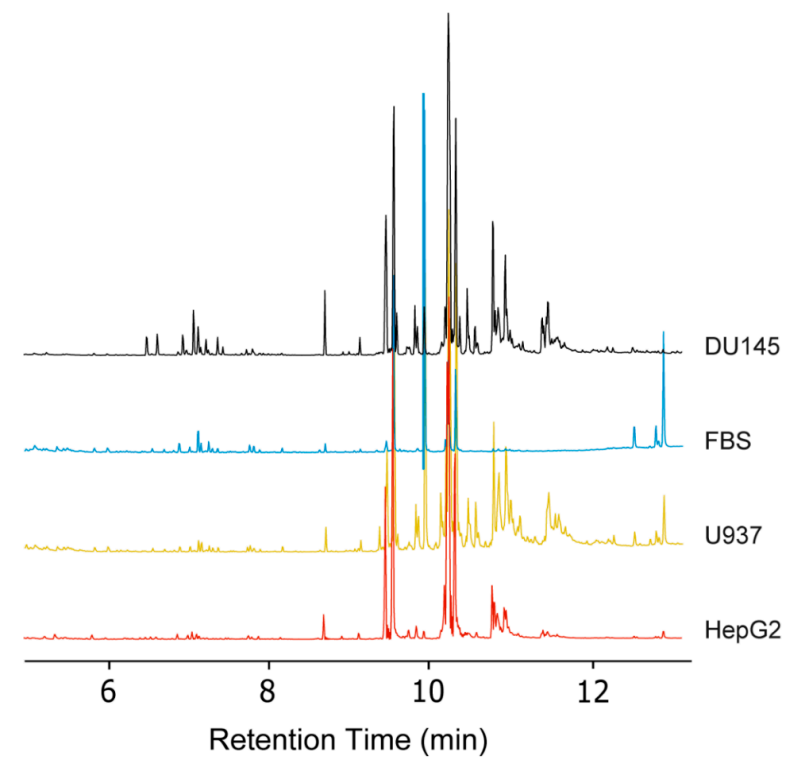

Figure 6. Chromatographs of FAMEs generated by TMSH derivatization. Representative chromatograms of biological matrices generated by TMSH derivatization, and the methods outlined in this paper. 


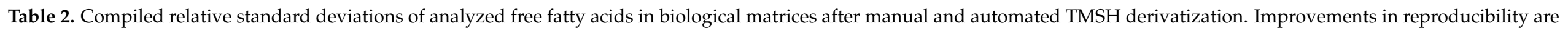
indicated with bolded \%RSD values. NF = not found.

\begin{tabular}{|c|c|c|c|c|c|c|c|c|c|}
\hline \multirow{2}{*}{ Fatty Acid } & \multirow{2}{*}{$\begin{array}{c}\text { Carbons:Double } \\
\text { Bonds }\end{array}$} & \multicolumn{2}{|c|}{ HepG2 n = 6} & \multicolumn{2}{|c|}{ DU145 n = 6} & \multicolumn{2}{|c|}{ U937 $n=6$} & \multicolumn{2}{|c|}{ FBS $n=6$} \\
\hline & & Manual & Automatic & Manual & Automatic & Manual & Automatic & Manual & Automatic \\
\hline Heptanoic acid & $\mathrm{C} 7: 0$ & NF & $\mathrm{NF}$ & $\mathrm{NF}$ & $\mathrm{NF}$ & $\mathrm{NF}$ & $\mathrm{NF}$ & NF & $\mathrm{NF}$ \\
\hline Octanoic acid & C8:0 & 12.29 & 6.74 & 10.80 & 5.16 & 7.88 & 5.33 & 24.24 & 18.95 \\
\hline Nonanoic acid & C9:0 & NF & NF & $\mathrm{NF}$ & NF & NF & NF & NF & NF \\
\hline Decanoic acid & C10:0 & 4.77 & 4.79 & 10.36 & 2.23 & 3.86 & 4.76 & 7.32 & 4.87 \\
\hline Undecanoic acid & C11:0 & 4.13 & 3.68 & NF & NF & NF & $\mathrm{NF}$ & 6.52 & 5.87 \\
\hline Dodecanoic acid & C12:0 & 3.33 & 2.55 & 5.29 & 2.94 & 3.15 & 4.48 & 7.18 & 5.63 \\
\hline Tridecanoic acid & C13:0 & 5.44 & 7.24 & NF & NF & 14.27 & 11.59 & 21.36 & 23.31 \\
\hline 9(Z)-Tetradecenoic acid & C14:1 & NF & NF & NF & NF & NF & NF & NF & NF \\
\hline Tetradecanoic acid & C14:0 & 5.23 & 5.43 & NF & NF & 3.02 & 4.06 & 8.70 & 5.09 \\
\hline 9(Z)-Pentedecenoic acid & C15:1 & 3.81 & 8.69 & 51.44 & 5.88 & 6.97 & 3.00 & 5.50 & 12.39 \\
\hline Pentadecanoic acid & C15:0 & 6.17 & 7.41 & 41.89 & 21.60 & 3.13 & 4.66 & 11.34 & 7.35 \\
\hline 9(Z)-Hexadecenoic acid & C16:1 & 3.37 & 3.19 & 30.09 & 17.32 & 2.26 & 1.82 & 19.98 & 7.27 \\
\hline Hexadecanoic acid & $\mathrm{C} 16: 0$ & 3.25 & 5.00 & 7.93 & 11.17 & 2.19 & 3.28 & 6.83 & 5.04 \\
\hline 10(Z)-Heptadecenoic acid & $\mathrm{C} 17: 1$ & 4.79 & 14.33 & NF & NF & 4.51 & 3.04 & 9.08 & 9.14 \\
\hline Heptadecanoic acid & $\mathrm{C} 17: 0$ & 6.49 & 3.79 & 19.65 & 7.05 & 1.00 & 1.03 & 4.69 & 2.69 \\
\hline$(6 Z, 9 Z, 12 Z)$-Octadecatrienoic acid & $\mathrm{C} 18: 3$ & NF & NF & NF & NF & 14.21 & 19.39 & NF & NF \\
\hline$(9 Z, 12 Z)$-octadecadienoic acid & $\mathrm{C} 18: 2$ & NF & NF & $\mathrm{NF}$ & NF & 5.42 & 2.74 & 20.74 & 5.43 \\
\hline Octadecanoic acid & C18:0 & 9.03 & 2.78 & 11.31 & 7.59 & 1.79 & 2.04 & 5.28 & 4.33 \\
\hline$(5 Z, 8 Z, 11 Z, 14 Z)$-Icosatetraenoic acid & C20:4 & 14.34 & 15.46 & 71.96 & 28.56 & 9.21 & 3.77 & NF & NF \\
\hline$(5 Z, 8 Z, 11 Z)$-Eicosatrienoic acid & C20:3 & 11.10 & 15.08 & 40.73 & 20.70 & 10.79 & 5.57 & NF & NF \\
\hline$(11 Z, 14 Z)$-Eicosadienoic acid & C20:2 & 7.70 & 12.23 & 18.63 & 12.29 & NF & NF & NF & NF \\
\hline 13(Z)-Eicosenoic acid & C20:1 & 6.19 & 12.72 & 11.22 & 4.70 & 7.81 & 4.96 & NF & NF \\
\hline Eicosanoic acid & C20:0 & 27.20 & 5.50 & 10.44 & 4.78 & 5.07 & 3.46 & 44.30 & 29.24 \\
\hline Heinecosanoic acid & C21:0 & NF & NF & NF & NF & NF & NF & NF & NF \\
\hline $\begin{array}{c}\text { (4Z, 7Z, 10Z, 13Z, 16Z, } \\
\text { 19Z)-Docosahexaenoic acid }\end{array}$ & $\mathrm{C} 22: 6$ & 22.28 & 14.46 & NF & $\mathrm{NF}$ & 6.48 & 5.94 & NF & NF \\
\hline (13Z)-Docosenoic acid & C22:1 & NF & NF & 11.85 & 9.28 & NF & $\mathrm{NF}$ & NF & NF \\
\hline Docosanoic acid & $\mathrm{C} 22: 0$ & 8.70 & 20.28 & 11.27 & 3.11 & 5.10 & 6.38 & 28.19 & 17.57 \\
\hline Tricosanoic acid & $\mathrm{C} 23: 0$ & NF & NF & 47.77 & 14.83 & 8.81 & 6.36 & NF & NF \\
\hline (15Z)-Tetracosenoic acid & $\mathrm{C} 24: 1$ & 8.30 & 14.30 & 12.74 & 6.92 & 8.66 & 3.37 & 42.72 & 6.93 \\
\hline Tetracosanoic acid & C24:0 & 6.59 & 33.25 & 4.77 & 4.48 & 8.20 & 2.44 & 44.78 & 7.33 \\
\hline Hexacosanoic acid & $\mathrm{C} 26: 0$ & 10.07 & 7.00 & 8.50 & 7.60 & 6.25 & 3.08 & 11.95 & 7.41 \\
\hline
\end{tabular}




\section{Materials and Methods}

\subsection{Chemicals}

Chemicals were purchased at the highest high-performance liquid chromatography (HPLC) purity grade available. HPLC-grade methanol, iso-octane, and methyl tert-butyl ether (MTBE) were purchased from Thermo Fisher Scientific (Waltham, MA). The TMSH solution was purchased from Sigma-Aldrich (St. Louis, MO, USA). The antioxidant 2,6-Ditert-butyl-4-methylphenol was purchased from Acros Organics (Geel, Belgium).

Analytical grade standards of fatty acids from 7 carbons of length to 26 were purchased to test the efficacy of TMSH derivatization in both automated and manual methodologies. Heptanoic (C7:0), nonanoic (C9:0), decanoic (C10:0), undecanoic (C11:0), tridecanoic (C13:0), pentadecanoic (C15:0), (Z)-hexadec-9-enoic(C16:1), heptadecanoic (C17:0), (6Z, 9Z, 12Z)-octadeca-6,9,12-trienoic (C18:3), (9Z, 12Z)-octadeca- 9,12-dienoic (C18:2), (Z)octadec-9-enoic (C18:1), nonadecanoic (C19:0), (5Z, 8Z, 11Z, 14Z, 17Z)-icosa-5,8,11,14,17pentaenoic (C20:5), (5Z, 8Z, 11Z, 14Z)-icosa-5,8,11,14-tetraenoic (C20:4), heinecosanoic (C21:0), (4Z, 7Z, 10Z, 13Z, 16Z, 19Z)-docosa-4,7,10,13,16,19-hexaenoic (C22:6), (13Z, 16Z)docosa- 13,16-dienoic (C22:2), (Z)-docos-13-enoic (C22:1), tricosanoic (C23:0), (Z)-tetracos15-enoic (C24:1), tetracosanoic (C24:0), and hexacosanoic (C26:0) acid standards were acquired from Supelco through Sigma-Aldrich (St. Lious, MO). Dodecanoic (C12:0), tridecanoic (C13:0), 9(Z)-tetradecenoic (C14:1), tetradecanoic (C14:0), 10(Z)-pentedecenoic (C15:1), pentadecanoic (C15:0), (9Z)-hexadecenoic (C16:1), hexadecenoic (C16:0), 10(Z)heptadecenoic (C17:1), heptadecanoic (C17:0), (6Z, 9Z, 12Z)-octadeca-6,9,12-trienoic (C18:3), (9Z, 12Z)-octadeca-9,12-dienoic (C18:2), (Z)-octadec-9-enoic(C18:1), octadecanoic (C18:0), 11(Z),14(Z),17(Z)-eicosatrienoic (C20:3), 11(Z),14(Z)-eicosadienoic (C20:2), 13(Z)-eicosenoic $(C 20: 1)$, eicosanoic (C20:0), and docosanoic (C22:0) acid standards were acquired from Cayman Chemical (Ann Arbor, MI, USA). Octanoic acid was acquired from MP Biochemicals (Irvine, CA, USA). All standards were injected and analyzed at a concentration of $110 \mu \mathrm{g} / \mathrm{mL}$.

\subsection{Biological Samples}

To validate this methodology, fatty acid standards and several sample matrices commonly used in research and clinical laboratories were derivatized, including three cell line extracts and characterized fetal bovine serum (FBS; Fischer Scientific, Hampton, NH, USA).

Three human cancer cell lines from the American Type Culture Collection (ATCC, Manasas, VA, USA) were selected to validate the derivatization method's efficacy on laboratory samples. The hepatocyte cancer cell line HepG2 was grown in Eagle's Minimum Essential Media (EMEM). The prostate and acute myeloid leukemia human cancer cell lines DU145 and U937 were cultured using Roswell Park Memorial Institute (RPMI) 1640 media. The cell culture media were modified to contain a $2 \mathrm{mM}$ glutamine final concentration and $10 \%$ FBS. Cell growth was maintained at $37^{\circ} \mathrm{C}$ and $5 \% \mathrm{CO}_{2}$. HepG2 cells were harvested when the cell count reached 30 million cells. The total cell extract was split into ten equal aliquots with 3 million cells in each aliquot. Cells were harvested and washed three times with ice-cold phosphate buffer saline. Excess liquid was removed as much as possible before the cell pellets were snap frozen in liquid nitrogen. DU145 and U937 non-polar extracts were pooled and aliquoted into 12 and 16 equal aliquots, respectively, with the cell counts being 30 and 50 million, respectively. All samples were stored at $-80^{\circ} \mathrm{C}$ until analysis.

\subsection{Lipid Extraction}

Previously frozen sera and cell pellets were thawed on ice before extraction, and the whole extraction process was performed at $4{ }^{\circ} \mathrm{C}$. The extraction of lipid molecules was accomplished by a modified Folch extraction that utilized HPLC-grade iso-octane as the non-polar phase instead of chloroform [24,53]. For serum samples, $50 \mu \mathrm{L}$ of serum was combined with $100 \mu \mathrm{L}$ of $0.9 \%$ saline and $200 \mu \mathrm{L}$ of HPLC-grade methanol, followed by $20 \mu \mathrm{L}$ of $1 \mathrm{~N} \mathrm{HCl}$ acid. Then, $20 \mu \mathrm{L}$ of internal standard deuterated heptadecanoic acid 
(C17:0) was added for a final concentration of $20 \mathrm{ug} / \mathrm{mL}$, and $10 \mu \mathrm{L}$ of $200 \mathrm{ug} / \mathrm{mL}$ of the antioxidant butylated hydroxytoluene (BHT) was added to the mixture to reach a final polar volume of $400 \mu \mathrm{L}$. Finally, $800 \mu \mathrm{L}$ of HPLC-grade iso-octane was added to each sample and vortexed for $30 \mathrm{~s}[54,55]$. After vortex mixing, samples were shaken for $10 \mathrm{~min}$ at $2500 \mathrm{rpm}$ on a Heidolph Vibramax 110 (Schwabach, Germany). Immediately after shaking, samples were centrifuged at $4750 \mathrm{rpm}$ for $20 \mathrm{~min}$ at a temperature of $4{ }^{\circ} \mathrm{C}$. The non-polar iso-octane supernatant was recovered using $500 \mu \mathrm{L}$ glass Hamiltonian syringes and transferred to a new $1.5 \mathrm{~mL}$ glass vial. These non-polar fractions were dried for $1.5 \mathrm{~h}$ at $4{ }^{\circ} \mathrm{C}$ in a Labconco Refrigerated Centrivap Concentrator coupled with Labconco $-84{ }^{\circ} \mathrm{C}$ Centrivap Cold Trap (Kansas City, MO, USA). Dried samples were stored at $-80{ }^{\circ} \mathrm{C}$ until analysis.

\subsection{Manual Derivatization}

The use of TMSH for manual derivatization was adapted from previously published methods with slight modifications $[43,56]$. Previously dried samples were grouped into batches of 6-8 samples. Each batch was derivatized manually by the addition of $30 \mu \mathrm{L}$ TMSH and $60 \mu \mathrm{L}$ MTBE into each sample vial. Each vial was vortexed for $30 \mathrm{~s}$ to achieve adequate mixing of reagents and then placed in the temperature-controlled sample tray attached to the Triplus RSH autosampler (Thermo Scientific, Waltham, MA, USA). Samples were set to incubate for $20 \mathrm{~min}$ inside the sample tray and maintained at a constant $4{ }^{\circ} \mathrm{C}$. After the 20 min incubation, the TriPlus RSH autosampler arm injected $3 \mu \mathrm{L}$ of each sample for analysis. FBS and HepG2 samples required an increase in the injection volume to achieve adequate signal stability. Each batch included an empty vial containing the mixed derivatizing agents to monitor for reagent contamination and account for reagent background readings.

\subsection{Automated Derivatization}

The Thermo Scientific Triplus RSH Sampling Workflow Editor Software (Thermo Scientific, Waltham, MA, USA) was used to program the automated derivatization method. The workflow editor allows for the adaptation of various modules and programmable steps to create an easily modifiable method to handle, derivatize, and inject samples in an online manner. This automated method utilizes the robotic Triplus RSH Autosampler to add reagents, vortex, and inject samples in an automated and online manner. QSertVials with fused glass inserts that contained dried samples were placed in the refrigerated sample box attached to the autosampler. The normal polytetrafluoroethylene (PTFE) screw caps were replaced with similar caps that contained a magnetic covering, enabling automated movement in conjunction with the magnetic syringe adaptor of the autosampler. A $100 \mu \mathrm{L}$ airtight syringe was used to add $60 \mu \mathrm{L}$ of MTBE to a sample vial. The syringe was then rinsed three times with $80 \mu \mathrm{L}$ of Optima HPLC-grade methanol before $30 \mu \mathrm{L}$ of TMSH was added to the sample vial. The syringe was rinsed again before the sample vial was moved to the attached vortex module. The sample vial was vortexed for $30 \mathrm{~s}$ at $1500 \mathrm{rpm}$ and then returned to the refrigerated sample tray and incubated for $20 \mathrm{~min}$ at $4{ }^{\circ} \mathrm{C}$. The $100 \mu \mathrm{L}$ syringe was undocked and replaced with a $10 \mu \mathrm{L}$ syringe used to inject up to $3 \mu \mathrm{L}$ of the derivatized sample into the GC-MS for immediate analysis.

\subsection{GC-MS Setup and Analysis}

The GC-MS system utilized is a Thermo Scientific Trace 1310 GC (Thermo Fisher Scientific, Waltham, MA, USA) coupled to an ISQ single quadrupole MS. The Triplus RSH autosampler is mounted to the system with several attached modules, including a temperature-controlled drawer for sample storage, a mounted vortex unit capable of mixing several sized sample vials, two large reagent reservoirs capable of containing $100 \mathrm{~mL}$ of chemical reagents or washing solutions, and an automated tool change (ATC) station to facilitate the use of multiple syringes. To enable the transport of sample vials between modules for automated derivatization, $9 \mathrm{~mm}$ magnetic AVCS screw caps from Thermo 
Scientific were needed and were used to seal the sample vials that undergo automated derivatization. Then, $3 \mu \mathrm{L}$ of each derivatized sample was injected into the GC-MS by the Triplus RSH autosampler. Samples were injected into a $4 \mathrm{~mm}$ glass split/splitless liner packed with quartz wool. The inlet was maintained at $250^{\circ} \mathrm{C}$, with a split ratio of 33.3 and a split flow of $55.0 \mathrm{~mL} / \mathrm{min}$ into a Phenomenex (Torrance, CA, USA) Zebron ZB 1-ms column. The column dimensions were the following: $30 \mathrm{~m}$ long, a $0.25 \mathrm{~mm}$ inner diameter, and a $0.25 \mu \mathrm{m}$ dimethylpolysiloxane stationary phase. Ultra-high purity helium from Praxair (Austin, TX, USA) was used as a carrier gas at a flow rate of $1.10 \mathrm{~mL} / \mathrm{min}$, and injection port septa were changed every 50 runs to minimize contamination between samples. The GC oven was held at the starting temperature of $80^{\circ} \mathrm{C}$ for two minutes and then set to increase $20^{\circ} \mathrm{C} / \mathrm{min}$ to $220^{\circ} \mathrm{C}$ and held there for $1 \mathrm{~min}$ before resuming the previous rate of $20^{\circ} \mathrm{C} / \mathrm{min}$ to a final temperature of $300{ }^{\circ} \mathrm{C}$. The oven was retained at $300{ }^{\circ} \mathrm{C}$ for five minutes to clean the column of residual cholesterol and its derivatives. The transfer line to the ISQ mass detector was kept at $280^{\circ} \mathrm{C}$, and the ion source was maintained at $230^{\circ} \mathrm{C}$. Ion scanning began $2.5 \mathrm{~min}$ after the initial injection and acquired ions in the mass range of 50-500 amu with a scan time of $0.5 \mathrm{~s}$. Ionization was accomplished through electron impact at $70 \mathrm{eV}$.

Individual fatty acids were confirmed by their corresponding standards' fragmentation patterns and retention times. The fragmentation pattern retention times were used to create a component-based processing method to extract ion areas for each confirmed fatty acid. Individual ion peak features were extracted by Xcalibur version 4.4, which utilized the ICIS peak detection algorithm with minimal smoothing and a maximum baseline of 10 scans to integrate the standard confirmed fatty acid peaks. Extracted ion peak area data were extracted and processed with MATLAB and R statistical functions to calculate averages, standard deviations, and relative standard deviations for each fatty acid and derivatization method reported below.

\section{Conclusions}

Previous studies automating metabolite extractions and derivatization have reported improved efficiency and reproducibility for metabolite analysis $[12,16,22,57,58]$. The advent of method editor software enables the rapid adaptation of simple sample preparation methods, such as the TMSH derivatization method detailed in this study. Automated TMSH derivatization reduced (i) researcher handling of samples and potential errors associated with manual derivatization handling and (ii) the total time needed for the analysis. Additionally, the data obtained from the automated TMSH derivatization of analytical standards showed improved data reproducibility compared to manually handled samples. When applied to biological matrices, the automated TMSH derivatization demonstrated similar reproducibility on most fatty acids analyzed across several cancer cell lines and serum samples. This automated derivatization methodology, although validated on a GC-MS system, could be implemented on any analytical platform with autosampler and vortex modules. This study presents the application and adaptation of a well-established fatty acid derivatization technique to be performed in an automated and online manner, reducing researcher-induced variability and improving data reproducibility while enabling high-throughput fatty acid profiling.

Author Contributions: Conceptualization, P.G. and S.T.; methodology, P.G., A.S.R. and J.C.; validation, P.G., A.S.R., J.C. and X.L.; formal analysis, P.G.; resources, P.G., X.L., J.C. and Y.B.H.; data curation, P.G.; writing, P.G.; reviewing and editing A.S.R., X.L., J.C., A.L. and S.T.; data visualization was performed by P.G.; supervision, S.T.; project administration, S.T.; funding acquisition, S.T. All authors have read and agreed to the published version of the manuscript.

Funding: This work was in part supported by grants from the University of Texas System (ST: STAR Award), NIH R01 CA2062101 and NIH R01 CA228404.

Institutional Review Board Statement: Not applicable.

Informed Consent Statement: Not applicable. 
Data Availability Statement: Compiled data is reported in the tables above. The raw data files are available from authors upon request.

Acknowledgments: Technical support and advice was provided by Thermo Fisher Scientific Employees Ada Powers and Amit Gujar.

Conflicts of Interest: The authors declare no conflict of interest.

Sample Availability: Samples of the compounds are not available from the authors. Fatty acid standards are available from the manufacturers listed in the materials and methods section.

\section{References}

1. Albergamo, A.; Rigano, F.; Purcaro, G.; Mauceri, A.; Fasulo, S.; Mondello, L. Free fatty acid profiling of marine sentinels by nanoLC-EI-MS for the assessment of environmental pollution effects. Sci. Total Environ. 2016, 571, 955-962. [CrossRef] [PubMed]

2. Song, E.M.; Byeon, J.-S.; Lee, S.M.; Yoo, H.J.; Kim, S.J.; Lee, S.-H.; Chang, K.; Hwang, S.W.; Yang, D.-H.; Jeong, J.-Y. Fecal fatty acid profiling as a potential new screening biomarker in patients with colorectal cancer. Dig. Dis. Sci. 2018, 63, 1229-1236. [CrossRef] [PubMed]

3. Abdelmagid, S.A.; Clarke, S.E.; Nielsen, D.E.; Badawi, A.; El-Sohemy, A.; Mutch, D.M.; Ma, D.W. Comprehensive profiling of plasma fatty acid concentrations in young healthy Canadian adults. PLoS ONE 2015, 10, e0116195. [CrossRef] [PubMed]

4. Wang, D.C.; Sun, C.H.; Liu, L.Y.; Sun, X.H.; Jin, X.W.; Song, W.L.; Liu, X.Q.; Wan, X.L. Serum fatty acid profiles using GC-MS and multivariate statistical analysis: Potential biomarkers of Alzheimer's disease. Neurobiol. Aging 2012, 33, 1057-1066. [CrossRef] [PubMed]

5. Ruiz-Nunez, B.; Dijck-Brouwer, D.A.; Muskiet, F.A. The relation of saturated fatty acids with low-grade inflammation and cardiovascular disease. J. Nutr. Biochem. 2016, 36, 1-20. [CrossRef]

6. Morris, G.; Berk, M.; Carvalho, A.; Caso, J.R.; Sanz, Y.; Walder, K.; Maes, M. The Role of the Microbial Metabolites Including Tryptophan Catabolites and Short Chain Fatty Acids in the Pathophysiology of Immune-Inflammatory and Neuroimmune Disease. Mol. Neurobiol. 2017, 54, 4432-4451. [CrossRef]

7. Manson, J.E.; Cook, N.R.; Lee, I.M.; Christen, W.; Bassuk, S.S.; Mora, S.; Gibson, H.; Albert, C.M.; Gordon, D.; Copeland, T.; et al. Marine n-3 Fatty Acids and Prevention of Cardiovascular Disease and Cancer. N Engl J. Med. 2019, 380, 23-32. [CrossRef] [PubMed]

8. Tiziani, S.; Kang, Y.; Harjanto, R.; Axelrod, J.; Piermarocchi, C.; Roberts, W.; Paternostro, G. Metabolomics of the tumor microenvironment in pediatric acute lymphoblastic leukemia. PLoS ONE 2013, 8, e82859. [CrossRef]

9. Zini, C.A.; Lord, H.; Christensen, E.; de, A.T.; Caramao, E.B.; Pawliszyn, J. Automation of solid-phase microextraction-gas chromatography-mass spectrometry extraction of eucalyptus volatiles. J. Chromatogr. Sci. 2002, 40, 140-146. [CrossRef]

10. Parkinson, D.R.; Bruheim, I.; Christ, I.; Pawliszyn, J. Full automation of derivatization-solid-phase microextraction-gas chromatography-mass spectrometry with a dual-arm system for the determination of organometallic compounds in aqueous samples. J. Chromatogr. A 2004, 1025, 77-84. [CrossRef] [PubMed]

11. Yang, L.; Lan, C.; Liu, H.; Dong, J.; Luan, T. Full automation of solid-phase microextraction/on-fiber derivatization for simultaneous determination of endocrine-disrupting chemicals and steroid hormones by gas chromatography-mass spectrometry. Anal. Bioanal. Chem. 2006, 386, 391-397. [CrossRef]

12. Hutchinson, J.P.; Setkova, L.; Pawliszyn, J. Automation of solid-phase microextraction on a 96-well plate format. J. Chromatogr. A 2007, 1149, 127-137. [CrossRef]

13. Zarate, E.; Boyle, V.; Rupprecht, U.; Green, S.; Villas-Boas, S.G.; Baker, P.; Pinu, F.R. Fully Automated Trimethylsilyl (TMS) Derivatisation Protocol for Metabolite Profiling by GC-MS. Metabolites 2016, 7, 1. [CrossRef]

14. Muller, Z.; Stelzl, E.; Bozic, M.; Haas, J.; Marth, E.; Kessler, H.H. Evaluation of automated sample preparation and quantitative PCR LCx assay for determination of human immunodeficiency virus type 1 RNA. J. Clin. Microbiol. 2004, 42, 1439-1443. [CrossRef]

15. Gu, Q.; David, F.; Lynen, F.; Rumpel, K.; Dugardeyn, J.; Van Der Straeten, D.; Xu, G.; Sandra, P. Evaluation of automated sample preparation, retention time locked gas chromatography-mass spectrometry and data analysis methods for the metabolomic study of Arabidopsis species. J. Chromatogr. A 2011, 1218, 3247-3254. [CrossRef]

16. Lerch, O.; Temme, O.; Daldrup, T. Comprehensive automation of the solid phase extraction gas chromatographic mass spectrometric analysis (SPE-GC/MS) of opioids, cocaine, and metabolites from serum and other matrices. Anal. Bioanal. Chem. 2014, 406, 4443-4451. [CrossRef] [PubMed]

17. Fiehn, O. Extending the breadth of metabolite profiling by gas chromatography coupled to mass spectrometry. Trends Anal. Chem 2008, 27, 261-269. [CrossRef]

18. Tsugawa, H.; Bamba, T.; Shinohara, M.; Nishiumi, S.; Yoshida, M.; Fukusaki, E. Practical non-targeted gas chromatography/mass spectrometry-based metabolomics platform for metabolic phenotype analysis. J. Biosci. Bioeng. 2011, 112, 292-298. [CrossRef]

19. Li, M.; Zhou, Z.; Nie, H.; Bai, Y.; Liu, H. Recent advances of chromatography and mass spectrometry in lipidomics. Anal. Bioanal. Chem. 2011, 399, 243-249. [CrossRef] [PubMed] 
20. Dunn, W.B.; Broadhurst, D.; Begley, P.; Zelena, E.; Francis-McIntyre, S.; Anderson, N.; Brown, M.; Knowles, J.D.; Halsall, A.; Haselden, J.N.; et al. Procedures for large-scale metabolic profiling of serum and plasma using gas chromatography and liquid chromatography coupled to mass spectrometry. Nat. Protoc. 2011, 6, 1060-1083. [CrossRef] [PubMed]

21. Mateo-Castro, R.; Gimeno-Adelantado, J.V.; Bosch-Reig, F.; Doménech-Carbó, A.; Casas-Catalán, M.J.; Osete-Cortina, L.; De la Cruz-Cañizares, J.; Doménech-Carbó, M.T. Identification by GC-FID and GC-MS of amino acids, fatty and bile acids in binding media used in works of art. Fresenius J. Anal. Chem. 2001, 369, 642-646. [CrossRef] [PubMed]

22. Abbiss, H.; Rawlinson, C.; Maker, G.L.; Trengove, R. Assessment of automated trimethylsilyl derivatization protocols for GC-MS-based untargeted metabolomic analysis of urine. Metabolomics 2015, 11, 1908-1921. [CrossRef]

23. Giovane, A.; Balestrieri, A.; Napoli, C. New insights into cardiovascular and lipid metabolomics. J. Cell. Biochem. 2008, 105, 648-654. [CrossRef]

24. Avula, S.G.C.; Belovich, J.M.; Xu, Y. Determination of fatty acid methyl esters derived from algae Scenedesmus dimorphus biomass by GC-MS with one-step esterification of free fatty acids and transesterification of glycerolipids. J. Sep. Sci. 2017, 40, 2214-2227. [CrossRef]

25. Cui, Y.; Chen, X.; Liu, L.; Xie, W.; Wu, Y.; Wu, Q.; Wang, D. Gas chromatography-mass spectrometry analysis of the free fatty acids in serum obtained from patients with Alzheimer's disease. Biomed. Mater. Eng 2015, 26, S2165-S2177. [CrossRef]

26. Han, S.; Karlowicz-Bodalska, K.; Potaczek, P.; Wojcik, A.; Ozimek, L.; Szura, D.; Musial, W. Identification of unknown impurity of azelaic acid in liposomal formulation assessed by HPLC-ELSD, GC-FID, and GC-MS. AAPS PharmSciTech 2014, 15, 111-120 [CrossRef]

27. Han, X.; Guo, J.; You, Y.; Yin, M.; Ren, C.; Zhan, J.; Huang, W. A fast and accurate way to determine short chain fatty acids in mouse feces based on GC-MS. J. Chromatogr. B Anal. Technol. Biomed. Life Sci. 2018, 1099, 73-82. [CrossRef]

28. Liu, Z.; Weng, R.; Feng, Y.; Li, Z.; Wang, L.; Su, X.; Yu, C. Fatty acid profiling of blood cell membranes by gas chromatography with mass spectrometry. J. Sep. Sci. 2016, 39, 3964-3972. [CrossRef] [PubMed]

29. Quehenberger, O.; Armando, A.M.; Dennis, E.A. High sensitivity quantitative lipidomics analysis of fatty acids in biological samples by gas chromatography-mass spectrometry. Biochim. Biophys. Acta 2011, 1811, 648-656. [CrossRef]

30. Argus, J.P.; Yu, A.K.; Wang, E.S.; Williams, K.J.; Bensinger, S.J. An optimized method for measuring fatty acids and cholesterol in stable isotope-labeled cells. J. Lipid Res. 2017, 58, 460-468. [CrossRef] [PubMed]

31. Halket, J.M.; Waterman, D.; Przyborowska, A.M.; Patel, R.K.; Fraser, P.D.; Bramley, P.M. Chemical derivatization and mass spectral libraries in metabolic profiling by GC/MS and LC/MS/MS. J. Exp. Bot. 2005, 56, 219-243. [CrossRef] [PubMed]

32. Furuhashi, T.; Weckwerth, W. Introduction to Lipid (FAME) Analysis in Algae Using Gas Chromatography-Mass Spectrometry. Handb. Plant. Metab. 2013, 215-225.

33. Fang, M.; Ivanisevic, J.; Benton, H.P.; Johnson, C.H.; Patti, G.J.; Hoang, L.T.; Uritboonthai, W.; Kurczy, M.E.; Siuzdak, G. Thermal Degradation of Small Molecules: A Global Metabolomic Investigation. Anal. Chem. 2015, 87, 10935-10941. [CrossRef] [PubMed]

34. Kang, J.X.; Wang, J. A simplified method for analysis of polyunsaturated fatty acids. BMC Biochem. 2005, 6, 1-4. [CrossRef]

35. Zhang, S.; Wang, H.; Zhu, M.J. A sensitive GC/MS detection method for analyzing microbial metabolites short chain fatty acids in fecal and serum samples. Talanta 2019, 196, 249-254. [CrossRef]

36. Lepage, G.; Roy, C.C. Direct transesterification of all classes of lipids in a one-step reaction. J. Lipid Res. 1986, 27, 114-120. [CrossRef]

37. Shin, H.-Y.; Lim, S.-M.; Bae, S.-Y.; Oh, S.C. Thermal decomposition and stability of fatty acid methyl esters in supercritical methanol. J. Anal. Appl. Pyrolysis 2011, 92, 332-338. [CrossRef]

38. Olivares-Carrillo, P.; Quesada-Medina, J. Thermal decomposition of fatty acid chains during the supercritical methanol transesterification of soybean oil to biodiesel. J. Supercrit. Fluids 2012, 72, 52-58. [CrossRef]

39. Firl, N.; Kienberger, H.; Hauser, T.; Rychlik, M. Determination of the fatty acid profile of neutral lipids, free fatty acids and phospholipids in human plasma. Clin. Chem. Lab. Med. 2013, 51, 799-810. [CrossRef]

40. Sutherland, K. Derivatisation using $\mathrm{m}$-(trifluoromethyl)phenyltrimethylammonium hydroxide of organic materials in artworks for analysis by gas chromatography-mass spectrometry: Unusual reaction products with alcohols. J. Chromatogr. A 2007, 1149, 30-37. [CrossRef]

41. Khakimov, B.; Motawia, M.S.; Bak, S.; Engelsen, S.B. The use of trimethylsilyl cyanide derivatization for robust and broadspectrum high-throughput gas chromatography-mass spectrometry based metabolomics. Anal. Bioanal. Chem. 2013, 405, 9193-9205. [CrossRef] [PubMed]

42. Yamauchi, K.; Tanabe, T.; Kinoshita, M. Trimethylsulfonium hydroxide: A new methylating agent. J. Org. Chem. 1979, 44, 638-639. [CrossRef]

43. Müller, K.-D.; Husmann, H.; Nalik, H.; Schomburg, G. Trans-esterification of fatty acids from microorganisms and human blood serum by trimethylsulfonium hydroxide (TMSH) for GC analysis. Chromatographia 1990, 30, 245-248. [CrossRef]

44. Müller, K.D.; Nalik, H.P.; Schmid, E.N.; Husmann, H.; Schomburg, G. Fast identification of mycobacterium species by GC analysis with trimethylsulfonium hydroxide (TMSH) for transesterification. J. High. Resolut. Chromatogr. 1993, 16, 161-165. [CrossRef]

45. Dron, J.; Linke, R.; Rosenberg, E.; Schreiner, M. Trimethylsulfonium hydroxide as derivatization reagent for the chemical investigation of drying oils in works of art by gas chromatography. J. Chromatogr. A 2004, 1047, 111-116. [CrossRef] 
46. Reinnicke, S.; Bernstein, A.; Elsner, M. Small and reproducible isotope effects during methylation with trimethylsulfonium hydroxide (TMSH): A convenient derivatization method for isotope analysis of negatively charged molecules. Anal. Chem. 2010, 82, 2013-2019. [CrossRef]

47. Butte, W.; Eilers, J.; Kirsch, M. Trialkylsulfonium- and Trialkylselenoniumhydroxides for the Pyrolytic Alkylation of Acidic Compounds. Anal. Lett. 1982, 15, 841-850. [CrossRef]

48. Ishida, Y.; Wakamatsu, S.; Yokoi, H.; Ohtani, H.; Tsuge, S. Compositional analysis of polyunsaturated fatty acid oil by one-step thermally assisted hydrolysis and methylation in the presence of trimethylsulfonium hydroxide. J. Anal. Appl. Pyrolysis 1999, 49, 267-276. [CrossRef]

49. Vosmann, K.; Klein, E.; Weber, N. Reaction of fatty acid amides and ethanolamides with trimethylsulfonium hydroxide. J. Chromatogr. A 1997, 773, 239-247. [CrossRef]

50. Koek, M.M.; Jellema, R.H.; van der Greef, J.; Tas, A.C.; Hankemeier, T. Quantitative metabolomics based on gas chromatography mass spectrometry: Status and perspectives. Metabolomics 2011, 7, 307-328. [CrossRef]

51. Chiu, H.H.; Tsai, S.J.; Tseng, Y.J.; Wu, M.S.; Liao, W.C.; Huang, C.S.; Kuo, C.H. An efficient and robust fatty acid profiling method for plasma metabolomic studies by gas chromatography-mass spectrometry. Clin. Chim. Acta 2015, 451, 183-190. [CrossRef]

52. Tan, B.; Zhang, Y.; Zhang, T.; He, J.; Luo, X.; Bian, X.; Wu, J.; Zou, C.; Wang, Y.; Fu, L. Identifying potential serum biomarkers of breast cancer through targeted free fatty acid profiles screening based on a GC-MS platform. Biomed. Chromatogr. 2020, 34 , e4922. [CrossRef] [PubMed]

53. Folch, J.; Lees, M.; Stanley, G.S. A simple method for the isolation and purification of total lipides from animal tissues. J. Biol. Chem. 1957, 226, 497-509. [CrossRef]

54. Li, Y.; Watkins, B.A. Analysis of Fatty Acids in Food Lipids. Curr. Protoc. Food Anal. Chem. 2001, 00, D1.2.1-D1.2.15. [CrossRef]

55. Barthet, V.; Chornick, T.; Daun, J. Comparison of Methods to Measure the Oil Contents in Oilseeds. J. Oleo Sci. 2002, 51, 589-597. [CrossRef]

56. Hosli, R.; Tobler, A.; Konig, S.; Muhlebach, S. A quantitative phenytoin GC-MS method and its validation for samples from human ex situ brain microdialysis, blood and saliva using solid-phase extraction. J. Anal. Toxicol 2013, 37, 102-109. [CrossRef] [PubMed]

57. Lerch, O.; Zinn, P.; Gotze, H.J. Automation of the GC/MS analysis of mineral oil contaminations in water. Fresenius J. Anal. Chem 2000, 367, 195-200. [CrossRef]

58. Shimbo, K.; Kubo, S.; Harada, Y.; Oonuki, T.; Yokokura, T.; Yoshida, H.; Amao, M.; Nakamura, M.; Kageyama, N.; Yamazaki, J.; et al. Automated precolumn derivatization system for analyzing physiological amino acids by liquid chromatography/mass spectrometry. Biomed. Chromatogr 2010, 24, 683-691. [CrossRef] 